\title{
Do we prescribe what patients prefer? Pilot study to assess patient preferences for medication regimen characteristics
}

This article was published in the following Dove Press journal:

Patient Preference and Adherence

24 September 2012

Number of times this article has been viewed

\author{
Diana Witticke ${ }^{1,2}$ \\ Hanna Marita Seidling ${ }^{1,2}$ \\ Hans-Dieter Klimm ${ }^{3}$ \\ Walter Emil Haefeli ${ }^{1,2}$ \\ 'Department of Clinical Pharmacology \\ and Pharmacoepidemiology, \\ ${ }^{2}$ Cooperation Unit Clinical Pharmacy, \\ ${ }^{3}$ Department of General Practice and \\ Health Service Research, University \\ of Heidelberg, Heidelberg, Germany
}

Correspondence: Walter E Haefeli Department of Clinical Pharmacology and Pharmacoepidemiology, Im Neuenheimer Feld 4I0,

Heidelberg 69120, Germany

Tel +4906221568740

$\mathrm{Fax}+4906221564642$

Email walter.emil.haefeli@med.uni-

heidelberg.de
Background: The aim of this pilot study was to evaluate patients' self-reported attitudes towards medication-related factors known to impair adherence and to assess their prevalence in ambulatory care as an essential prerequisite to improve patient adherence.

Methods: We conducted a face-to-face interview with 110 primary care patients maintained on at least one drug. For each drug, the patient was asked to specify medication-related factors of interest, ie, dosage form, dosage interval, required relationship with food intake, and the planned time of day for intake, and to rate the individual relevance of each prevalent parameter on a three-point Likert scale (discriminating between prefer, neutral, and dislike).

Results: Tablets with a once-daily dosage frequency were the most preferred dosage form, with a high prevalence in the ambulatory setting. Drug intake in the morning and evening were most preferred, and drug intake at noon was least preferred, but also had a low prevalence in contrast with drug intake independent of meals that was most preferred. Interestingly, only one quarter (26.4\%) of all the patients were able to indicate clear preferences or dislikes.

Conclusion: When patients are asked to specify their preferences for relevant medication regimen characteristics, they clearly indicated regimens that have been associated with better adherence in earlier studies. Therefore, our results suggest that adaptation of drug regimens to individual preferences might be a promising strategy to improve adherence. Because the German health care system may differ from other systems in relevant aspects, our findings should be confirmed by evaluation of patient preferences in other health care systems. Once generalizability of the study results is shown, these findings could be a promising basis upon which to promote patient adherence right from the beginning of drug therapy.

Keywords: patient perception, medication regimen complexity, adherence

\section{Introduction}

Patient adherence with drug treatment is an important predictor of the success of drug therapy. ${ }^{1-3}$ However, a multitude of factors related to the patient, medication, disease (eg, symptomatology), provider (eg, patient-provider relationship), and health care system (eg, copayment) may impair adherence and consequently impact successful drug treatment. ${ }^{4,5}$ Patient-related factors reducing adherence with drug therapy include a decline in cognitive function, skepticism about the benefit of long-term treatment and its consequences on health, and low health literacy. ${ }^{6-9}$ Medication-related factors with a negative impact on patient adherence involve multiple dosages per day, ${ }^{10-21}$ certain dosage forms, ${ }^{22,23}$ additional handling directions like tablet splitting, ${ }^{22,24}$ and dependency on food intake. ${ }^{11,12,14}$ Hence, medication-related factors might be particularly promising targets for strategies that aim at improving patient adherence 
because many of them can be rather easily modified and corrected. ${ }^{25}$ However, not all of these characteristics are barriers to treatment success in all patients and, interestingly, pill burden per se is not a relevant barrier in all patients, so smooth integration of the regimen into the daily activities of the individual may be more important. ${ }^{26}$ Therefore, when eliminating problematic medication-related characteristics, patient preferences should also be considered. Indeed, patient preferences are particularly important during shared decision-making, a technique that successfully increases patient adherence and improves the clinical outcome. ${ }^{27,28}$ Most studies assessing patient preferences have focused on the decision to start or not to start a drug therapy due to potential harm (eg, medication side effects) or benefit (eg, effectiveness for specific symptoms). ${ }^{27-29}$ Hence, the aim of this study was to evaluate how patients perceive different medication-related factors and to assess the prevalence of these factors in ambulatory care as an essential prerequisite for a successful intervention.

\section{Materials and methods}

The study was performed between January 28, 2011 and February 4, 2011 in ambulatory patients who were predominantly accessed via one large private practice in Germany. After approval by the ethics committee of the Medical Faculty of Heidelberg, we conducted a face-to-face interview with 110 ambulatory care patients taking at least one long-term medication (ie, prescription of at least one drug for the treatment of a chronic disorder). During the interview, we noted for each patient on an anonymized documentation sheet the number of prescription and nonprescription drugs and the total duration for which the patient had taken at least one drug. For each drug, the patient was asked to specify the medication-related factors of interest, ie, dosage form, dosage interval, required relationship with food intake, and the time of day the drug was supposed to be taken. Thereby, we evaluated the prevalence of each factor. We excluded all details with a prevalence $<10 \%$ from the interpretation; however, we kept them for illustration purposes in the respective figures. In addition to collection of objective medication-related information, we also surveyed the individual appraisal of each prevalent parameter on a three-point Likert scale (discriminating between prefer, neutral, and dislike). In addition to analysis of the whole data set, patients were allocated into two groups, ie, those who either did or did not express clear preferences in the majority of responses. For this purpose, we arbitrarily defined patients with clear preferences as those who gave $<20 \%$ neutral responses and all others as patients without clear preferences. Because of the anonymous nature of the interview, we did not capture demographic characteristics, such as patient age and gender, and did not record individual drugs.

\section{Statistical analysis}

All data were analyzed descriptively and reported as proportions and means, including standard deviations. Prevalence was calculated as the proportion of patients with a distinct medication-related characteristic, with medication-related characteristics having low prevalence (ie, $<10 \%$ ) being excluded. Preference was calculated as the proportion of patients who preferred the respective characteristic of all patients with the respective medication-related characteristic. Preferences of different medication-related characteristics were compared by Chi-square test. Statistical significance was accepted at $P<0.05$. All analyses were conducted using SPSS version 19 (IBM ${ }^{\circledR}$ SPSS $^{\circledR}$ Statistics, Armonk, NY).

\section{Results}

We interviewed 110 primary care patients with at least one long-term treatment about their drug use. Most of the patients $(\mathrm{n}=82,74.5 \%)$ had taken their medication for at least 6 years, with 58 patients $(52.7 \%)$ having taken their medication for more than 10 years. The mean number of drugs was $4.0 \pm 1.9$ and comprised $3.2 \pm 1.7$ prescription and $0.9 \pm 0.9$ nonprescription drugs, with $58.2 \%$ of patients taking at least one nonprescription drug. Five patients did not specify their drug count, except for indicating that it comprised $>10$ drugs. These patients were excluded from the analyses assessing drug numbers.

To estimate the validity of the statements made by the patients, we conducted a separate analysis to evaluate whether the views of patients with clear preferences differed from those in the overall population. Of all 110 patients, $29(26.4 \%)$ were able to indicate clear preferences or dislikes relating to dosage frequencies, dosage forms, times of day, and drug-food relationships in $80 \%$ of their responses. Most patients $(n=69,62.7 \%)$ indicated clear preferences only for some of the medication-related characteristics, and in some patients $(n=12,10.9 \%)$, the neutral ranking was most prevalent.

\section{Frequency and preference of different dosage frequencies}

Once-daily drug intake was the most common dosage frequency $(91.8 \%)$, and preferred by the majority $(79.2 \%)$ 
of the overall study population. Preferences decreased with increasing number of daily applications, with no preference for three times daily drug applications. Nine times more patients preferred once-daily application compared with more frequent applications $(P<0.001)$. Dosage frequencies like "every three days" or "once a week" were also highly valued by the patients ( $100 \%$ and $60 \%$, respectively); however, their prevalence was very low $(0.9 \%$ and $4.5 \%)$. A separate analysis of results for patients who were able to indicate clear preferences (Figure 1A) revealed higher proportions of patients who preferred a once-daily or twicedaily dosage frequency than patients who did not indicate clear preferences $(P<0.001$, Figure 1B).

\section{Frequency and preference of different dosage forms}

Tablets were the most prevalent $(97.3 \%)$ and also the preferred dosage form (68.2\%) in the overall study population. Creams/ointments and capsules were also applied by more than $20 \%$ of the patients ( $24.5 \%$ and $21.8 \%$, respectively). However, there was a significantly higher preference for capsules than for creams or ointments $(54.2 \%$ versus $25.9 \%, P<0.001)$. Also, there was a high preference for syrup and patches (100\% and $66.7 \%)$, but these were
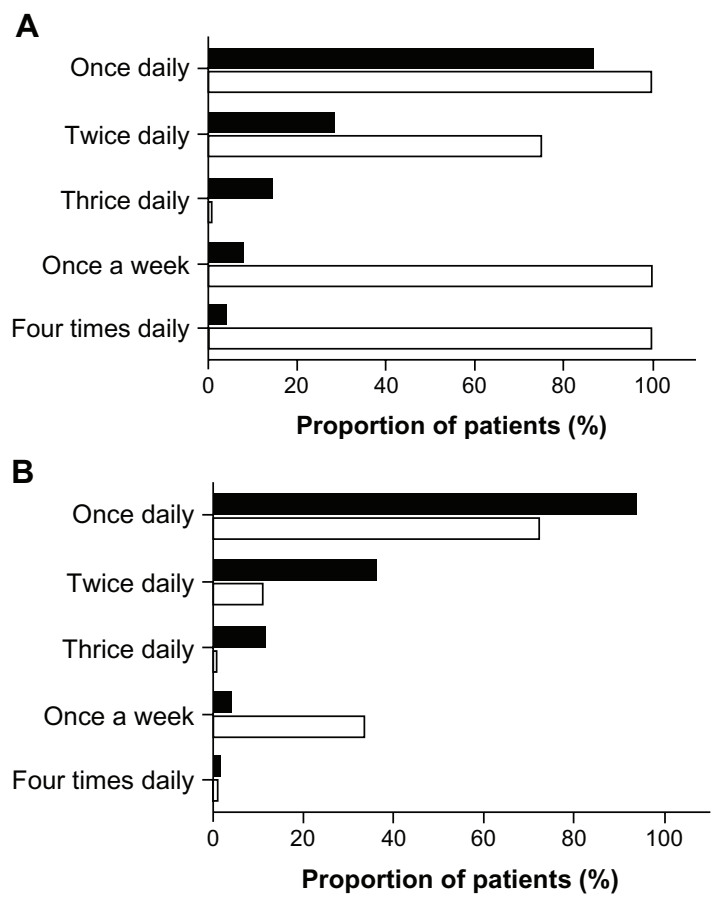

Figure I (A) Frequency $(\square)$ and preference $(\square)$ of different dosage frequencies as expressed by 29 general practice patients expressing clear views and preferences for most characteristics assessed. (B) Frequency ( $\square$ ) and preference ( $\square$ ) of different dosage frequencies as expressed by $8 \mathrm{I}$ general practice patients without an ability to express clear views and preferences for most characteristics assessed.
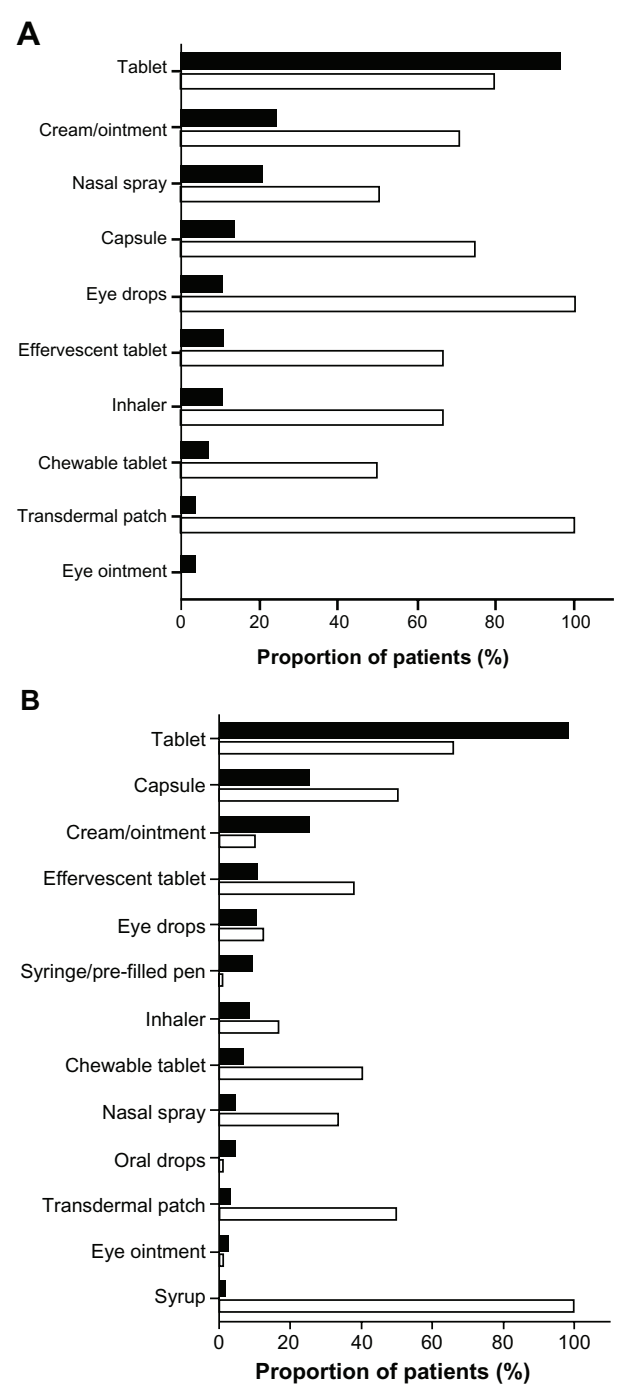

Figure 2 (A) Frequency ( $\square$ ) and preference ( $\square$ ) of different dosage forms as expressed by 29 general practice patients expressing clear views and preferences for most characteristics assessed. (B) Frequency ( $\square$ ) and preference ( $\square$ ) of different dosage forms as expressed by $8 \mathrm{I}$ general practice patients without an ability to express clear views and preferences for most characteristics assessed.

prescribed only rarely (for $0.9 \%$ and $2.7 \%$ of patients, respectively). The dosage forms with the lowest preference were eye ointments, drops for oral use, and prefilled pens for injection (not preferred by any patient) but their prevalence was very low $(<10 \%)$. In contrast, patients with clear preferences also rated eye drops as a preferred dosage form (Figure 2A). Differences in ratings of dosage forms between patients with or without clear preferences are shown in Figure 2A and B.

\section{Frequency and preference of different times of day for drug application}

Most of the patients in this study population had to take their drugs in the morning (94.5\%), and half of them preferred to 
take their drugs at that time of day. Drug intake in the evening was also common (66.4\%) and was the time of the day preferred by one quarter of the patients, but drug intake in the morning had higher patient acceptance $(P<0.001)$. Within our study population, drug intake at night showed the highest preference $(62.5 \%)$ but with low prevalence $(7.3 \%)$. The time of day with the lowest patient preference was noon $(11.8 \%$, prevalence $30.9 \% ; P=0.001$ ) compared with all other time points. The ratings were similar in the subgroups of patients with or without clear preferences (Figure 3A and B).

\section{Prevalence and preference of different drug-food relationships}

Most patients in the overall study population preferred to take drugs independently of meals $(75.0 \%$, prevalence $32.7 \%$ ) or after meals $(56.6 \%$, prevalence $48.2 \%)$. The lowest preference was given to medication taken 30 minutes before meals $(17.8 \%$, prevalence $40.9 \%)$. However, patients with clear preferences rated drug intake 30 minutes before meals and during meals with the same preference and showed some differences compared with the subgroup of patients without clear preferences (Figure 4A and B).
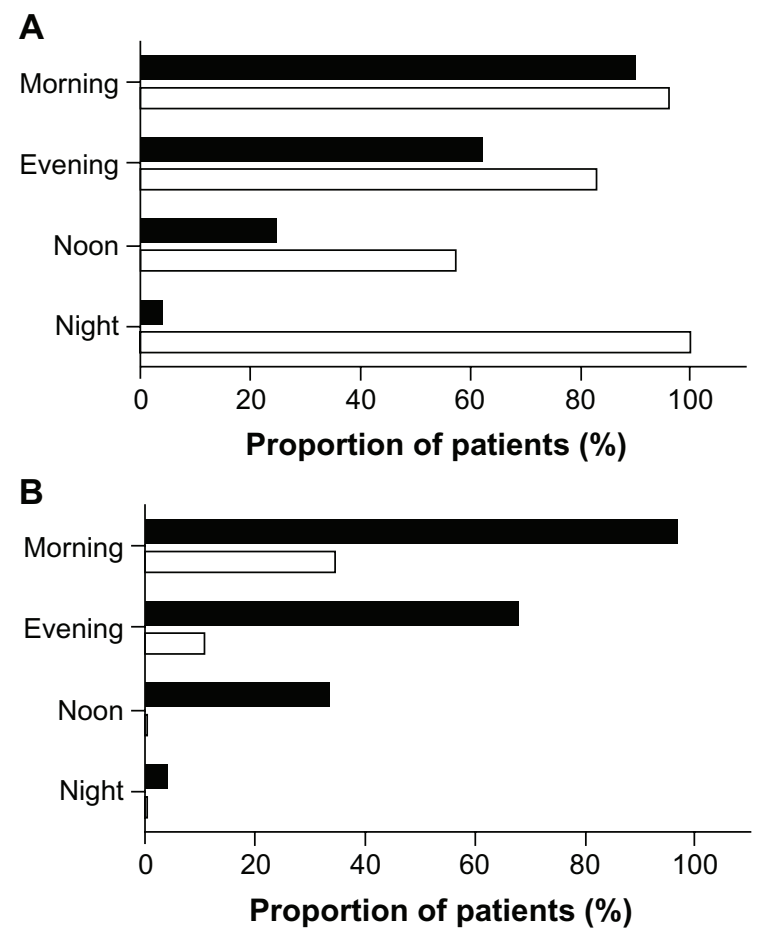

Figure 3 (A) Frequency ( $\square$ ) and preference $(\square)$ of different times of the day for drug application as expressed by 29 general practice patients expressing clear views and preferences for most characteristics assessed. (B) Frequency ( $\mathbf{\square})$ and preference $(\square)$ of different times of the day for drug application as expressed by 81 general practice patients without an ability to express clear views and preferences for most characteristics assessed.
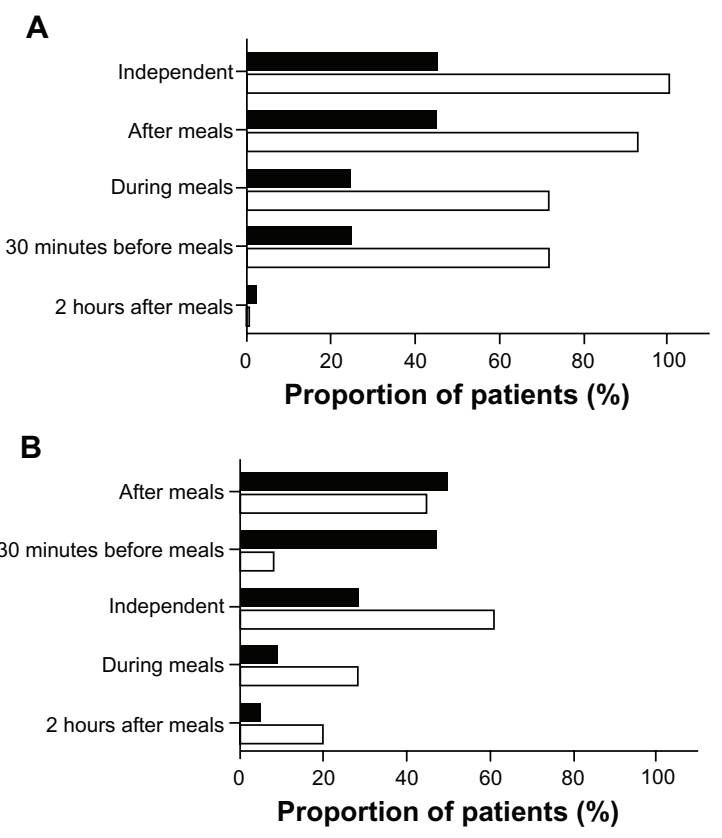

Figure 4 (A) Frequency $(\square)$ and preference $(\square)$ of different drug-food relationships expressed by 29 general practice patients expressing clear views and preferences for most characteristics assessed. (B) Frequency $(\square)$ and preference $(\square)$ of different drug-food relationships expressed by 81 general practice patients without an ability to express clear views and preferences for most characteristics assessed.

\section{Discussion}

Active patient involvement into the treatment process (shared decision-making) is critical to the success of treatment, ${ }^{30}$ and consideration of patient preferences is an essential element to foster feasibility of a treatment, its acceptance, and ultimately adherence with drug therapy. ${ }^{31,32}$ Therefore, we evaluated patient attitudes towards different medicationrelated factors and assessed their prevalence in ambulatory care. Tablets with a once-daily administration scheme were most frequently prescribed and also the patients' preferred dosage form and frequency. The clear preference for oncedaily administration confirmed the results of earlier studies showing that adherence decreases with increasing number of daily drug administrations, ${ }^{10,15,16,21,33}$ and thus emphasizes the impact of dosage frequency on adherence. Importantly, simplification of multidose prescriptions to a once-daily dosing regimen is possible in $18 \%$ of all cases, and is thus a promising strategy for optimizing patient preference. ${ }^{25}$ In addition, drug intake at noon was least accepted, confirming earlier findings of lower adherence with drug intake at this time of day. ${ }^{15}$ Also, rigid schedules of drug intake and meals (such as application 30 minutes before meals or 2 hours after meals) expectedly had only low acceptance rates.

From a theoretical point of view, patient preferences may have many different causes. First, patients might prefer specific medication-related characteristics because they seem 
associated with a drug effect. For instance, asthma patients like inhalers to the same extent as oral dosage forms (tablets or capsules) $)^{34}$ and because medication-related characteristics and perceived effect are closely and likely positively related, this is likely not an unbiased assessment. Second, patient preference might also result from better tolerability of drugs ${ }^{35}$ For instance, many patients preferred drug administration after meals. This might result from perceived and often documented better tolerability of drugs if taken after meals. On the other hand, drug administration independent of meals was clearly preferred. For many patients, the preferred method of drug intake is linked to characteristics that best fit into their daily life and do not inflict any constraints on them, ${ }^{35-37}$ and developing routines for self-management of medication is very challenging for patients. ${ }^{38}$ If drugs can be taken independent of meals, no special considerations are required.

In our patient population, only $26.4 \%$ indicated clear preferences for most medication-related characteristics. The results for this subgroup mostly reflect the results of the overall study population, but in a more distinct way. Divergent rankings occasionally occurred, eg, regarding preferences for drug intake, in that while the study population overall preferred drug intake during meals, patients with preferences ranked drug intake half an hour before meals equally high. Because patient preferences may modulate adherence to drug treatment, ${ }^{33,39}$ patients should be encouraged to express their preferences based on different factors concerning lifestyle and physical skills. For example, it may be critical to adapt drug intake as much as possible to their daily habits and to select the most convenient medication regimen, application technique, or dosage forms, but must also consider relevant limitations, such as dysphagia. ${ }^{40}$

Furthermore, in addition to modification by patient preferences, patient adherence is determined by a multiplicity of factors alone or in combination, including the patientprovider relationship, patient characteristics, the clinical setting, or the disease itself. ${ }^{41}$ Therefore, assessment of patient preferences and incorporation of drug therapy in the daily routine is only one method of optimizing patient adherence and should be repeatedly evaluated to detect and consider changes in daily routine or behavior.

This study has several limitations. Because of our anonymized study design, we did not document patient characteristics, including age, gender, or comorbidities. Therefore, we could not evaluate the impact of patient-related characteristics on preferences for different medication-related issues. Furthermore, we interviewed only a small albeit unselected population of primary care patients. Therefore, some medication-related characteristics applied only to a small group of patients and, consequently, preferences and prevalence could have been overestimated or underestimated. Further, we only included patients with at least one long-term treatment. Therefore, patients who stopped medication therapy due to dislike of certain medication regimen characteristics would not have been captured by this approach. Finally, in our survey, we combined creams and ointments because patients are often not familiar with the difference. Obviously, the properties of these dosage forms differ, so patient preferences may also differ. For these reasons, our study provides only preliminary evidence on these dosage forms.

\section{Conclusion}

Only a small proportion of ambulatory patients are able to indicate clear preferences with regard to medication-related characteristics. Therefore, patients should be encouraged and empowered to express their preferences as a prerequisite for shared decision-making and active participation in the treatment process. In this study, patients who were able to indicate their preferences clearly opted for regimens that had been associated with better adherence in earlier studies. Therefore, these results suggest that adaptation of drug regimens to individual preferences might be a promising strategy to improve adherence. Because the German health care system may differ from other systems in relevant aspects, our findings should be confirmed by evaluation of patient preferences in other health care systems. Once generalizability of the study results is shown, these findings could be a promising basis to promote patient adherence right from the beginning of drug therapy.

\section{Acknowledgment}

This project was supported in part by a grant from the Federal Ministry of Education and Research of Germany (BMBF 01GK0801).

\section{Disclosure}

The authors report no conflict of interest in this work.

\section{References}

1. Al-Qazaz H, Sulaiman SA, Hassali MA, et al. Diabetes knowledge, medication adherence and glycemic control among patients with type 2 diabetes. Int J Clin Pharm. 2011;33(6):1028-1035.

2. Dragomir A, Cote R, Roy L, et al. Impact of adherence to antihypertensive agents on clinical outcomes and hospitalization costs. Med Care. 2010; 48(5):418-425.

3. Kaiser RM, Schmader KE, Pieper CF, Lindblad CI, Ruby CM, Hanlon JT. Therapeutic failure-related hospitalisations in the frail elderly. Drugs Aging. 2006;23(7):579-586.

4. Osterberg L, Blaschke T. Adherence to medication. N Engl J Med. 2005; 353(5):487-497.

5. Ickovics JR, Meade CS. Adherence to HAART among patients with HIV: breakthroughs and barriers. AIDS Care. 2002;14(3):309-318. 
6. Gellad WF, Grenard JL, Marcum ZA. A systematic review of barriers to medication adherence in the elderly: looking beyond cost and regimen complexity. Am J Geriatr Pharmacother. 2011;9(1):11-23.

7. Huas D, Debiais F, Blotman F, et al. Compliance and treatment satisfaction of post menopausal women treated for osteoporosis. Compliance with osteoporosis treatment. BMC Womens Health. 2010;10:26.

8. Insel K, Morrow D, Brewer B, Figueredo A. Executive function, working memory, and medication adherence among older adults. J Gerontol B Psychol Sci Soc Sci. 2006;61(2):P102-P107.

9. Kripalani S, Gatti ME, Jacobson TA. Association of age, health literacy, and medication management strategies with cardiovascular medication adherence. Patient Educ Couns. 2010;81(2):177-181.

10. Paes AH, Bakker A, Soe-Agnie CJ. Impact of dosage frequency on patient compliance. Diabetes Care. 1997;20(10):1512-1517.

11. Chesney MA. Factors affecting adherence to antiretroviral therapy. Clin Infect Dis. 2000;30 Suppl 2:S171-S176.

12. Stone VE, Hogan JW, Schuman P, et al. Antiretroviral regimen complexity, self-reported adherence, and HIV patients' understanding of their regimens: survey of women in the her study. J Acquir Immune Defic Syndr. 2001;28(2):124-131.

13. Corsonello A, Pedone C, Lattanzio F, et al. Regimen complexity and medication nonadherence in elderly patients. Ther Clin Risk Manag. 2009;5(1):209-216.

14. Ammassari A, Trotta MP, Murri R, et al. Correlates and predictors of adherence to highly active antiretroviral therapy: overview of published literature. J Acquir Immune Defic Syndr. 2002;31 Suppl 3:S123-S127.

15. Eisen SA, Miller DK, Woodward RS, Spitznagel E, Przybeck TR. The effect of prescribed daily dose frequency on patient medication compliance. Arch Intern Med. 1990;150(9):1881-1884.

16. Claxton AJ, Cramer J, Pierce C. A systematic review of the associations between dose regimens and medication compliance. Clin Ther. 2001; 23(8):1296-1310.

17. Maitland D, Jackson A, Osorio J, Mandalia S, Gazzard BG, Moyle GJ. Switching from twice-daily abacavir and lamivudine to the once-daily fixed-dose combination tablet of abacavir and lamivudine improves patient adherence and satisfaction with therapy. HIV Med. 2008;9(8): 667-672.

18. Cramer JA, Mattson RH, Prevey ML, Scheyer RD, Ouellette VL. How often is medication taken as prescribed? A novel assessment technique. JAMA. 1989;261(22):3273-3277.

19. McDonald HP, Garg AX, Haynes RB. Interventions to enhance patient adherence to medication prescriptions: scientific review. JAMA. 2002;288(22):2868-2879.

20. Kardas P. Compliance, clinical outcome, and quality of life of patients with stable angina pectoris receiving once-daily betaxolol versus twice daily metoprolol: a randomized controlled trial. Vasc Health Risk Manag. 2007;3(2):235-242.

21. Saini SD, Schoenfeld P, Kaulback K, Dubinsky MC. Effect of medication dosing frequency on adherence in chronic diseases. Am J Manag Care. 2009;15(6):e22-e33.

22. Lam PW, Lum CM, Leung MF. Drug non-adherence and associated risk factors among Chinese geriatric patients in Hong Kong. Hong Kong Med J. 2007;13(4):284-292.

23. Buysman E, Conner C, Aagren M, Bouchard J, Liu F. Adherence and persistence to a regimen of basal insulin in a pre-filled pen compared to vial/syringe in insulin-naive patients with type 2 diabetes. Curr Med Res Opin. 2011;27(9):1709-1717.

Patient Preference and Adherence

\section{Publish your work in this journal}

Patient Preference and Adherence is an international, peer-reviewed, open access journal focusing on the growing importance of patient preference and adherence throughout the therapeutic continuum. Patient satisfaction, acceptability, quality of life, compliance, persistence and their role in developing new therapeutic modalities and compounds to
24. Hixson-Wallace JA, Dotson JB, Blakey SA. Effect of regimen complexity on patient satisfaction and compliance with warfarin therapy. Clin Appl Thromb Hemost. 2001;7(1):33-37.

25. Witticke D, Seidling HM, Lohmann K, Send AFJ, Haefeli WE. Opportunities to reduce medication regimen complexity: a retrospective analysis of 500 patients at discharge from internal medicine of a university hospital. Drug Saf. 2012. In press.

26. Gifford AL, Bormann JE, Shively MJ, Wright BC, Richman DD, Bozzette SA. Predictors of self-reported adherence and plasma HIV concentrations in patients on multidrug antiretroviral regimens. J Acquir Immune Defic Syndr. 2000;23(5):386-395.

27. Fried TR, Bradley EH, Towle VR. Assessment of patient preferences: integrating treatments and outcomes. J Gerontol B Psychol Sci Soc Sci. 2002;57(6):S348-S354.

28. Hauber AB, Mohamed AF, Johnson FR, Falvey H. Treatment preferences and medication adherence of people with Type 2 diabetes using oral glucose-lowering agents. Diabet Med. 2009;26(4):416-424.

29. Van Brunt K, Matza LS, Classi PM, Johnston JA. Preferences related to attention-deficit/hyperactivity disorder and its treatment. Patient Prefer Adherence. 2011;5:33-43.

30. Say RE, Thomson R. The importance of patient preferences in treatment decisions - challenges for doctors. BMJ. 2003;327(7414):542-545.

31. Loh A, Simon D, Wills CE, Kriston L, Niebling W, Harter M. The effects of a shared decision-making intervention in primary care of depression: a cluster-randomized controlled trial. Patient Educ Couns. 2007;67(3):324-332.

32. Joosten EA, DeFuentes-Merillas L, de Weert GH, Sensky T, van der Staak CP, de Jong CA. Systematic review of the effects of shared decision-making on patient satisfaction, treatment adherence and health status. Psychother Psychosom. 2008;77(4):219-226.

33. Granger AL, Fehnel SE, Hogue SL, Bennett L, Edin HM. An assessment of patient preference and adherence to treatment with Wellbutrin SR: a web-based survey. J Affect Disord. 2006;90(2-3):217-221.

34. Gillissen A, Lecheler J. Bronchial asthma: a comparison of the doctor's assessment and the patient's opinion. Dtsch Med Wochenschr. 2004;129(10):484-489. German.

35. Emkey R, Koltun W, Beusterien K, et al. Patient preference for oncemonthly ibandronate versus once-weekly alendronate in a randomized, open-label, cross-over trial: the Boniva Alendronate Trial in Osteoporosis (BALTO). Curr Med Res Opin. 2005;21(12):1895-1903.

36. Haynes RB, Sackett DL, Gibson ES, et al. Improvement of medication compliance in uncontrolled hypertension. Lancet. 1976;1(7972): $1265-1268$

37. Logan AG, Milne BJ, Achber C, Campbell WP, Haynes RB. Work-site treatment of hypertension by specially trained nurses. A controlled trial. Lancet. 1979;2(8153):1175-1178.

38. Haslbeck JW, Schaeffer D. Routines in medication management: the perspective of people with chronic conditions. Chronic Illn. 2009;5(3):184-196.

39. Reginster JY, Rabenda V, Neuprez A. Adherence, patient preference and dosing frequency: understanding the relationship. Bone. 2006; 38(4 Suppl 1):S2-S6.

40. Carnaby-Mann G, Crary M. Pill swallowing by adults with dysphagia. Arch Otolaryngol Head Neck Surg. 2005;131(11):970-975.

41. Ickovics JR, Meisler AW. Adherence in AIDS clinical trials: a framework for clinical research and clinical care. J Clin Epidemiol. 1997;50(4):385-391.

optimize clinical outcomes for existing disease states are major areas of interest. This journal has been accepted for indexing on PubMed Central. The manuscript management system is completely online and includes a very quick and fair peer-review system. Visit http://www.dovepress.com/ testimonials.php to read real quotes from published authors. 